Review article

\title{
A review of accelerator-based searches for Neutral Long-Lived Particles
}

\section{Una revisión de las búsquedas de partículas neutras de larga vida de experimentos basados en aceleradores}

\author{
(1D) Marta Losada \\ Division of Science, New York University Abu Dhabi, Saadiyat Island, Abu Dhabi, UAE \\ Possession article for admission as a member corresponding to the \\ Academia Colombiana de Ciencias Exactas, Físicas y Naturales
}

\begin{abstract}
In this paper we present the current status of searches for neutral long-lived particles. The basic formalism that allows the determination of the number of expected long-lived particles is presented. Heavy neutral leptons can be a type of long-lived particles. The main observational motivations for the existence of heavy neutral lepton is covered as well. A summary of the main results from both collider searches and fixed target/beam dump experiments is presented. The outlook for next generation experiments and their impact on the parameter space of coupling strength and mass of heavy neutral leptons is also discussed.
\end{abstract}

Keywords: Long-lived particles; collider searches; fixed target meson decays; heavy neutral leptons.

\begin{abstract}
Resumen
En este artículo se presenta una revisión de la búsqueda de partículas neutras con tiempo de vida largos. El formalismo esencial que determina el número de partículas con tiempos de vida largos se presenta. Leptones neutros pesados pueden ser un tipo de partícula con tiempo de vida largo. Un resumen de los resultados principales de búsquedas de colisionadores y de blanco fijo son presentados. Se discute también la nueva generación de experimentos y su impacto en el espacio de parámetros relevante de la intensidad de acoplamiento y masa de los leptones neutros pesados.
\end{abstract}

Palabras clave: partículas de larga vida; búsquedas de colisionadores; decaímientos de mesones en blancos fijos; leptones neutros pesados.

\section{Introduction}

The Large Hadron Collider (LHC) has been in the 21st century the ultimate high energy physics machine. It can operate as a proton-proton collider and it delivered up to $2018147 \mathrm{fb}^{-1}$ integrated luminosity, Aad, et al. (2019a). The success of the LHC is overwhelming with the discovery of the Higgs boson in 2012, Chathrchya, et al. (2012), Aad, et al. (2012). As a result of Run I, with full luminosity, a precise measurement of the Higgs boson mass of, $\mathrm{mh}=$ $125.09+/-0.21$ (stat) +/- 0.11 (sys) GeV was obtained, and the production modes were also confirmed to be essentially Standard Model (SM)-like, Aad, et al. (2015). The focus of Run II was the measurement of the Higgs boson couplings, to gauge bosons, quarks and leptons, another fantastic achievement of the LHC machine and experiments, Aad, et al. (2016). In addition, at the increased center of mass energy of Run II (13 TeV), very exhaustive and relevant electroweak measurements were performed confirming once again the SM predictions, Aaboud, et al. (2019c), Sirunyan, et al. (2019a).

There are, however, very strong motivations for searching for new physics at the LHC and other experiments, as the SM cannot provide adequate solutions to fundamental questions such as: dark matter, neutrino masses, matterantimatter asymmetry, etc.

Dedicated searches have been performed for direct production of dark matter, supersymmetry, and many other exotic searches of extensions of the SM. Up until now

\footnotetext{
Corresponding autor:

Marta Losada; malosada@uan.edu.co

Received: June 16, 2019

Accepted: October 7, 2019

Editor: María Elena Gómez
} 
exclusions on significant regions of parameter space for the Beyond the Standard Model (BSM) models invoked to solve the aforementioned problems have been established, see references, Aaboud, et al. (2019d), Sirunyan, et al. (2019b) for the exclusion plots.

An intriguing possibility of BSM physics are longlived particles (LLPs). The theoretical motivations for these LLPs arise from various type of models including: Supersymmetric-like models, or Gauge, Higgs, Neutrino, Vector, Axion portals. These portals or dark-sides are essentially the mechanism in which a specific SM particle will couple with the particle content of the dark side. Typically, they are weakly coupled and/or there are available ranges of mass that allow them to have long life times, Alimena, et al. (2019), Lee, et al. (2019).

In this case the main issues associated to the production and decay of LLPs are:

- Small number of events

- Novel decay signatures requiring a significant deviation from standard analyses

- Modified Background contributions

Long-lived particles can be neutral or charged (electromagnetically or coloured). However, the only way to directly detect a neutral LLP is through its decay products. A recent effort has been made to determine possible complementary experiments and analyses that could also be performed to detect LLPs in the still available regions of parameter space. This includes new detectors strategically located with respect to the interaction points (IP) of the LHC detectors hoping to cover even longer decay lengths of the candidate LLPs, as well as, new fixed target and beam dump experiments. We focus on the case of neutral LLPs that are weakly interacting in this paper.

The main purpose of this article is to review the recent results and current status of experimental results from accelerator-based experiments, i.e. the LHC experiments and other currently running fixed target and/or beam dump experiments. In addition, a summary of the proposals for future experiments sensitive to LLPs is presented, Beacham, et al. (2019)].

The paper is organized as follows. Section 1 describes the main observational motivations for a specific type of possible LLP, the so-called heavy neutral leptons. Section 2 briefly presents the main aspects to be considered for the analysis of LLPs production and decays, specializing to a production mode via a parent particle for clarity and in order to show the specific details of the exclusion regions in parameter space. Section 3 reviews the main results from Run II at the LHC experiments as well as the latest constraints from fixed target or beam dump experiments. Section 4 focuses on the outlook on a 10 to 15 -year timescale of new experiments or detectors that can increase the reach and sensitivity to LLPs. In section 5 we present the main conclusions.

\section{Observational motivations for the existence of Heavy Neutral Leptons}

We now detour briefly to consider current experimental observations that provide well motivated reasons for the existence of additional sterile states in addition to the Standard Model particle content.

Models of neutrino masses. Solar, atmospheric and reactor neutrino experiments all indicate the existence of non-zero neutrino masses Esteban (2017). Theoretical motivations for generating neutrino masses include diverse extensions of the Standard Model, such as incorporating sterile neutrinos, Dirac or Majorana, invoking a left-right asymmetry, grand unified theories and many more models, for a review see Hernandez (2015).

We consider the simplest extension of adding sterile neutrinos and recognizing the impact of the additional terms in the Lagrangian due to the presence of these new sterile states, Majorana (1937).

The Lagrangian is given by, Majorana (1937):

$$
\mathcal{L}=\mathcal{L}_{S M}-\sum^{n_{R}} \bar{l}_{L}^{\alpha} Y^{\alpha i} \tilde{\Phi} \nu_{R}^{i}-\sum^{n_{R}} \frac{1}{2} \bar{\nu}_{R}^{i c} M_{N}^{i j} \nu_{R}^{j}+\text { h.c. }
$$

Where, in addition to the Standard Model term, the second term is a Yukawa-type coupling between the sterile state, the left-handed lepton doublet and the Higgs boson. The third term is a mass term for the right-handed neutrino. Given this structure of the terms in the Lagrangian, a mixing between active and sterile neutrinos is now present.

One of the main aspects of course is the generation of masses for the left-handed neutrinos. On the other hand, charged and neutral currents containing neutrinos are now modified by the presence of this active-sterile mixing. The consequences of this modification of the currents is explored in detail in the accelerator-based experiments described below. Additionally, the Higgs invisible branching ratio to neutrino states is modified. There exist new additional phases in the lepton sector of the Standard Model when the additional sterile neutrinos are Majorana particles.

Leptogenesis. Another exciting possibility that is viable upon the introduction of extra sterile neutrinos states, is the dynamical generation of the matter - antimatter asymmetry that is observed in the Universe. Fukugita and Yanagida (1986), FY, were the first to propose the possibility of producing leptogenesis via lepton number violating decays of the right-handed neutrinos in a $\mathrm{CP}$ violating way in the backdrop of the expanding Universe. The net lepton asymmetry is then converted to a baryon asymmetry via rapid B-L conserving sphaleron processes before the electroweak phase transition occurs in the early Universe. Thus, fulfilling the necessary conditions formulated by Sakharov for producing dynamically the matter-antimatter asymmetry. Typically, the right-handed neutrinos are quite massive, on the order of $10^{10} \mathrm{GeV}$ in mass, with order (1) couplings. The mechanism of FY has undergone exhaustive 
investigations and significant variants have been identifiedsee references Davidson, et al. (2008), Nardi, et al. (2006), Abada, et al. (2006), Bhupal et al (2018). One of the most interesting variants proposed by Akhmedov, Rubakov and Smirnov, Akhmedov, et al. (1998) relies on generating the asymmetry via oscillations in the right handed neutrino sector, this coupled with flavor effects (whose relevance was determined in the works of Nardi, et al. (2006) and Abada, et al. (2006), can provide an alternative mechanism for leptogenesis with sterile neutrinos on the order of the MeV-GeV scale, Drewes, et al. (2018), Shaposhnikov, M (2008), Hernandez, et al. (2016). It is out of the scope of this paper to detail the mechanism and results of leptogenesis but suffice it to say that one of the main features is that the range of masses and mixings of the sterile states now lies within the range of accessibility of current and proposed experiments unlike the case of the FY-inspired models. This makes the search for these new heavy neutral leptons all the more exciting. The additional CP phases in the lepton sector are also a crucial ingredient for the mechanism to work and can provide novel signatures at experiments.

Dark matter. Observational astrophysics provides the main justification for the existence of dark matter Zwicky (1933). The particle physics interpretation of dark matter requires the existence of very weakly interacting particles that exist in the Universe, Bergstrom (2012). Sterile neutrinos of a given mass and couplings are still a viable solution to the dark matter problem Boyarsky, et al. (2012).

We do not delve into the details of this but simply state that in order to solve neutrino masses, leptogenesis and dark matter a minimum of three sterile neutrinos is required Asaka, et al. (2005). Two of these right-handed neutral leptons must be much heavier that the sterile neutrino that provides a solution to the dark matter abundance in the Universe.

\section{Basics of LLP production and decay}

There have been many searches for heavy neutral leptons over the last decades, figure 1 is a schematic representation of the reach in the relevant parameter space of a series of experiments that have carefully deployed searches for these extra particles, either via fixed target experiments, beam dump or collider experiments. For the fixed target/beam dump experiments the mass of the decaying hadron defines the energy reach, for colliders it is defined by the center of mass energy.

At colliders, the LLPs can be produced via one of the following processes: resonance, pair production, parent decay, Higgs boson decay, charged current. This is the case of the energy frontier in which it is through the direct production or parent particle production which is of a large mass that the sensitivity to LLP production is reached.

There are several scenarios for the decay of the LLP:

- It can decay to SM particles in the detector (analysis of final states)
- Its decay length can exceed the detector volume (missing energy )

On the other hand, in the intensity frontier, the aim is for larger rates with less backgrounds, although limited in energy reach. For fixed target /beam dump experiments long lived particles will be produced in the leptonic or semileptonic decays of mesons produced by the impinging protons. The mass of these mesons defines the maximum energy reach. In this case the LLP options are:

- It can decay before or in the detector to SM particles

- Its decay length can be larger than the detector region (missing energy)

- It can scatter with the shielding before the detector

Production. The total number of expected events with a heavy neutral lepton, HNL, decaying to SM particles is given by:

$$
\begin{aligned}
& \mathrm{N}^{\text {expected }}=\mathrm{L} \int \mathrm{d} \sigma \text { X BR (parent particle->LLP) } \\
& \text { X BR (LLP-> SM) X P_N X } \varepsilon \_ \text {geo }
\end{aligned}
$$

Where $\mathrm{L}$ is the total integrated luminosity, $\mathrm{d} \sigma$ the differential cross section, and the branching ratios from the parent particle to the LLP and from the LLP to the SM particles. $\mathrm{P} \_\mathrm{N}$ is the probability that the decay will occur within the detector volume, a measure of longitudinal acceptance, and $\varepsilon \_$geo is the geometrical acceptance.

It is then crucial to enhance the number of observed events considering the strong dependence on the size of the detector, the location with respect to the production of the LLP and the particle identification of the decay products.

We take as an example the case of Heavy Neutral Leptons. Depending on the production process the cross section that produces the LLP must be determined.

Consider the simple model of the Standard Model plus one right-handed neutrino given by the Lagrangian in eq. (1), presented in Section 1.

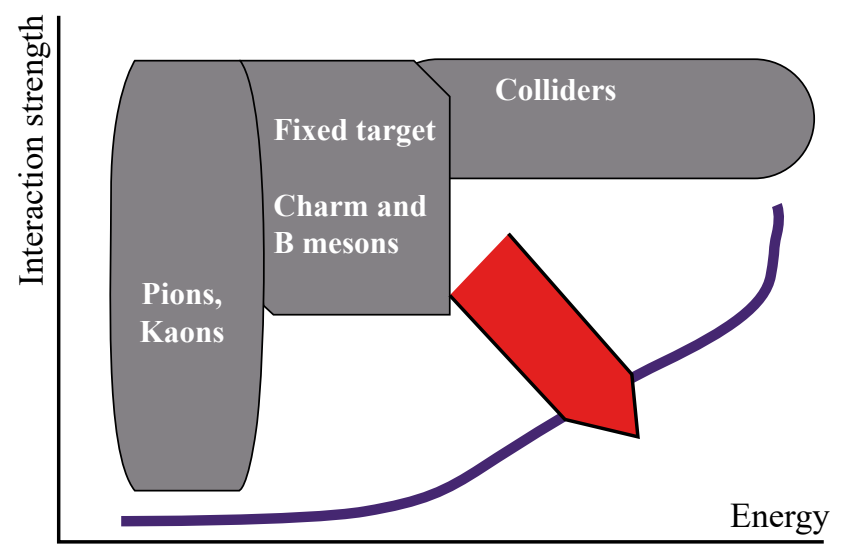

Figure 1. Schematic representation of exclusion regions from collider and fixed target/beam dump experiments in the plane of interaction strength versus energy associated to the LLP. The goal of new experiments is to continue to explore the rest of parameter space 
The LHC is a W boson factory, with an integrated luminosity of $3000 \mathrm{fb}^{-1}$, on the order of $10^{11} \mathrm{~W}$ bosons are expected to be produced from pp collisions. We consider the case of $\mathrm{M}_{\mathrm{N}}<\mathrm{m}_{\mathrm{w}}$.In this case the production of HNLs from $\mathrm{W}$ boson decay is flavor dependent. Instead the production via $\mathrm{Z}$ or $\mathrm{H}$ decay is flavor independent. Figure 2 shows the branching ratio of the gauge bosons $\mathrm{W}, \mathrm{Z}$ and the Higgs boson to the HNL, N. in the case of the simplest model of the SM plus one HNL, N.

Alternatively, the HNL can be produced at fixed target/ beam dump experiments from pions, kaons or charm and $\mathrm{B}$ meson decays, $\mathrm{M}$-> Nl, or M' -> M Nl.

Decay. In both cases, collider and fixed target experiments, the produced heavy neutral lepton will then decay. In the case of HNLs produced by mesons the maximum mass of the $\mathrm{N}$ is given by the kinematic limit. The decay of the $\mathrm{HNL}$, once again is mainly into leptonic or semileptonic final states and can be lepton number conserving or not. The lifetime will scale as, Gronau, et al. (1984):

$$
\mathrm{T} \sim \mathrm{G}_{\mathrm{F}}^{-2} \mathrm{M}_{\mathrm{N}}^{-5}|\mathrm{~V}|^{-2}
$$

In the case of colliders, for masses in the range $5 \mathrm{GeV}$ $30 \mathrm{GeV}$ the produced HNL can be long-lived and decay a measurable distance from the interaction point (IP). The smaller the mass and the mixing angle the longer the lifetime and decay length. The distance it travels is given by,

$$
\mathrm{L}=\mathrm{p} \mathrm{c} \mathrm{T}_{\mathrm{N}} / \mathrm{M}_{\mathrm{N}}
$$

As long as $\mathrm{V}^{2}$ is small enough (on the order of $10^{-6}$, for the range of masses we are interested in $(5-30 \mathrm{GeV})$, the lifetime and decay length will be large enough to be able to detect the particle. The probability that the LLP will decay in a specific detector volume is given by

$$
\mathrm{P}_{\mathrm{N}}=\exp \left(-\mathrm{D}_{1} / \mathrm{L}\right)-\exp \left(-\mathrm{D}_{2} / \mathrm{L}\right)
$$

Where $\mathrm{D}_{1}$ and $\mathrm{D}_{2}$ are given by the location and size of the detector with respect to the production point, Gronau, et al. (1984). The relevant point is that for as a function of the decay length $\mathrm{L}$, and the values of $\mathrm{D}_{1}$ and $\mathrm{D}_{2}$ it is possible to define a longitudinal acceptance of the detector.

Signatures. One of the characteristics of LLPs are their distinctive signatures in colliders that include: disappearing tracks, displaced multitrack vertices, displaced leptons, lepton-jets or lepton pairs, displaced and emerging jets, etc.

Although the HNL will decay to SM particles, the precise signatures will be different than the standard signatures expected at collider experiments. HNLs can produce displaced vertices, one of the key smoking gun signatures. At the secondary vertex the HNL decays to SM particles. However, in the case of colliders the precise features of the decay, no originating track for charged leptons and quarks, the low momentum of the outgoing particles, the small angle between the decay products if the HNL is highly boosted, etc make triggering and particle identification an important challenge.

If the HNL is a Majorana particle its decays can be lepton number conserving (LNC) or lepton number violating (LNV). There are two distinctive final states when the HNL decays after production through a W. First, consider the final state of dilepton and 2 jets. In this case, the mass of HNL can be fully reconstructed from the particles emanating from the displaced vertex. The leptons can be opposite sign (LNC) or same sign (LNV). The other final state to consider is a trilepton with missing energy, in this case the mass of the HNL cannot be fully reconstructed.

There are clear challenges for these signatures of the decay related to: displaced vertex reconstruction, track reconstruction efficiency, trigger acceptance and thresholds, background estimation and understanding.

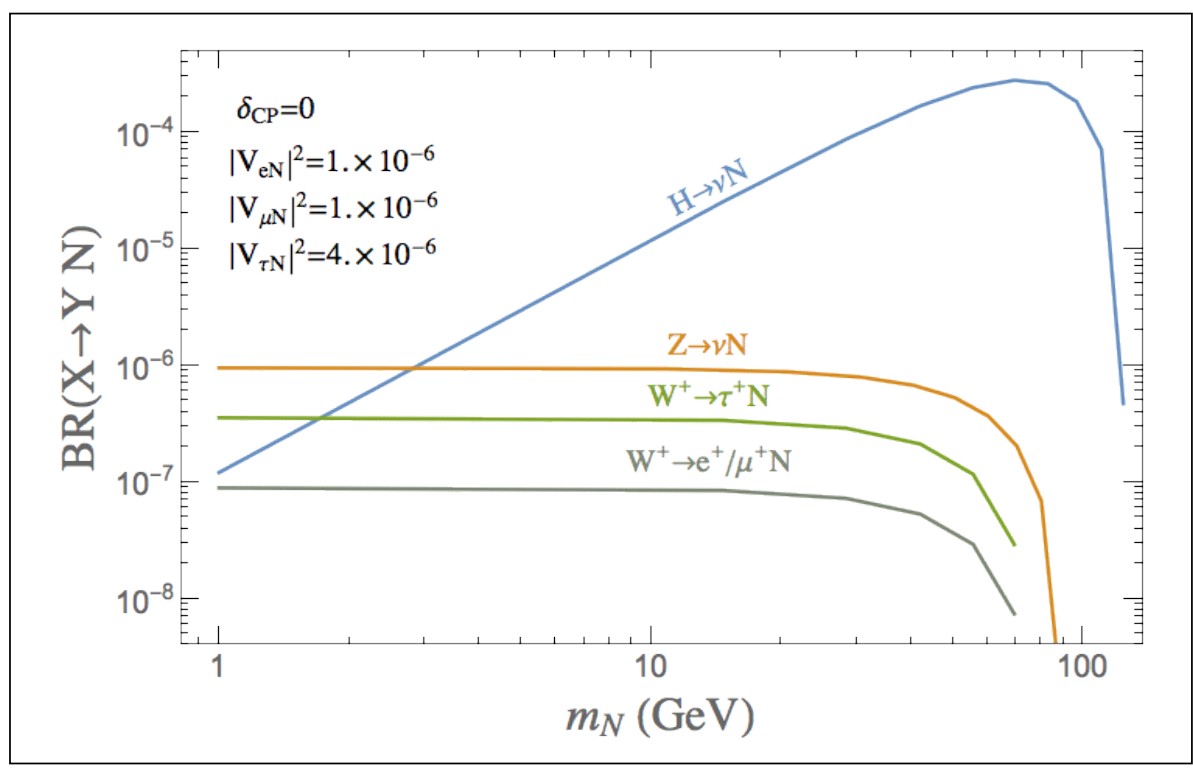

Figure 2. Branching ratios of $\mathrm{W}, \mathrm{Z}$ and $\mathrm{H}$ bosons to the heavy neutral lepton $\mathrm{N}$ in the $\mathrm{SM}+1 \mathrm{~N}$ model 
Background. For the signatures mentioned in the previous section the main backgrounds are provided by: random track crossings, delayed decays of SM particles, cosmic rays, hadronic interactions, etc.

The cosmic ray and the decays from boosted SM particles are reducible backgrounds that can be effectively reduced with appropriate cuts (reconstructed mass, angular separation, pseudo-rapidity). For the random track crossing a tight lepton id requirement and timing window with respect to bunch crossing is effective.

\section{Summary of main recent results}

There have been some results of searches on the context of HNLs performed at colliders and fixed target experiments that we summarize in this section.

ATLAS has performed a search for heavy neutral leptons with masses below the $\mathrm{W}$ boson mass. It separates the analysis into two regimes depending on the promptness of the decay of the right-handed neutrinos. It focuses on fully leptonic final states, considering both lepton number conserving and lepton number violating final states.

The results present for the first time the exclusion limits on displaced decays to lepton in the inner tracker of the ATLAS detector improving existing limits by one order of magnitude Aaboud, et al. (2019b). For displaced decays, signal strengths up to $210^{-6}$ are excluded for mass ranges in the $4.5-10 \mathrm{GeV}$ range.

Both ATLAS and CMS have provided limits for the region of prompt decays with fully leptonic decays in the final state. The main consequence is a small increment in the exclusion limit with respect to the mixing angle for the range of masses explored by the LEP experiments and an increase in the mass exclusion limit for larger masses that the range covered by LEP, Sirunyan, et al. (2018), Aaboud, et al. (2019b). ATLAS excludes coupling strengths larger the 4 $10^{-5}$ for the mass range $10-50 \mathrm{GeV}$. CMS has excluded signal intensities between $1.210^{-5}$ and 1,8 for masses between $1 \mathrm{GeV}$ and $1.2 \mathrm{TeV}$.

1. Over the last few decades there have been a plethora of experiments that have studied rare decays of pions, kaons and charmed mesons Bernardi, et al. (1988), Vaitaitis, et al. (1999), Bergsma, et al. (1986), Vilain, et al. (1995), Badier, et al. (1986), Artamonov, et al. (2015), Baranov, et al. (1993), Cooper-Sarkar, et al. (1986), Gallas, et al. (1995), Astier, et al. (2001), Aaji, et al. (2017), Aguilar-Arevalo, et al. (2015), Abreu, et al. (1997), Adriani, et al. (1992), Achard, et al. (2001), Aad, et al. (2015), Khachatryan, et al. (2015). All of these experiments have produced an exclusion region in the $|\mathrm{V}|^{2}$ versus $\mathrm{M}_{\mathrm{N}}$ plane.

PIENU. PIENU is a high precision experiment of rare decays at TRIUMF. Its focus is measuring lepton flavour universality in pion decays. This recent experiment has searched for peaks in the distribution of pion decays, from stopped pions of a pion beam of $75-\mathrm{MeV}$, increasing the exclusion limit below the pion mass in the range of mixing angle of the right-handed neutrino with the electron neutrino. The improvement factors in the exclusion are approximately an order of magnitude in the mixing angle with respect to previous searches in the same low mass $\mathrm{M}_{\mathrm{N}}$ range.

NA62. More recently, NA62, a fixed target experiment running at CERN, has produced two relevant results in the search of HNLs, Lazzeroni, et al. (2017), Cortina Gil, et al. (2019). This fixed target experiment copiously produces kaons. Thus, it provided an ideal setting for exploring the existence of HNLs in kaons decays. A recent result is the study of K-> N 1 process, increasing the exclusion region. In addition, NA62 has searched for LNC and LNV violating decays of kaons. A new limit is set on the branching ratios:

$$
\begin{aligned}
\mathcal{B}\left(K^{+} \rightarrow \pi^{-} e^{+} e^{+}\right) & <2.2 \times 10^{-10} \\
\mathcal{B}\left(K^{+} \rightarrow \pi^{-} \mu^{+} \mu^{+}\right) & <4.2 \times 10^{-11}
\end{aligned}
$$

In addition to target mode, NA62 can also run beam dump mode producing $\mathrm{D}$ and $\mathrm{B}$ mesons that can then decay via the weak interactions to HNLs. The projected run for 2021-2023 could then set an improvement of bound obtained from the CHARM experiment of close to one order of magnitude.

Heavy ion collisions. Recently, a new analysis has been put forward that studies the case of searching for HNLs in heavy ion collisions at the LHC, Drewes, et al. (2019). The analysis considers the reach in $\mathrm{Pb}-\mathrm{Pb}$ and $\mathrm{Ar}-\mathrm{Ar}$ collisions versus p-p collisions in the decays of both $\mathrm{W}$ bosons and $\mathrm{B}$ meson decays. They conclude that although there is no increase in the exclusion limit in the case of $\mathrm{W}$ decays from heavy ion collisions versus proton-proton interactions. For the case of B meson decays in Ar-Ar collisions a significant increase of sensitivity is obtained, of two orders of magnitude in the upper bound of the mixing angle.

The HL-LHC upgrade will provide new opportunities to search for HNLs, although with some increased challenges in terms of the possible increased backgrounds for existing detectors CMS, ATLAS and LHCb, Alimena, et al. (2019).

\section{New proposals for extending LLP searches}

There are several recent proposals that have been made to increase the reach of the search for LLPs. Here we consider a few of them in detail, the reach in terms of exclusion plot for all is summarized in figure 3 in the plane of mixing angle squared versus right-handed neutrino mass.

FASER. FASER is a proposal that was approved in March 2019 to place a detector in the forward direction of an interaction point (IP) of the LHC (480m) and take advantage of the particles that are copiously produced due to the inelastic scattering, Ariga el at (2018), Feng et al (2018). These particles are mainly mesons that can decay to the LLP, as in the fixed target experiments. The detector is some distance away from the interaction point such that for long decay lengths the probability of actual decay of the LLP occurring in the detector will be enhanced. The main advantage are the negligible backgrounds and specially designed triggers sensitive to low momentum particles 


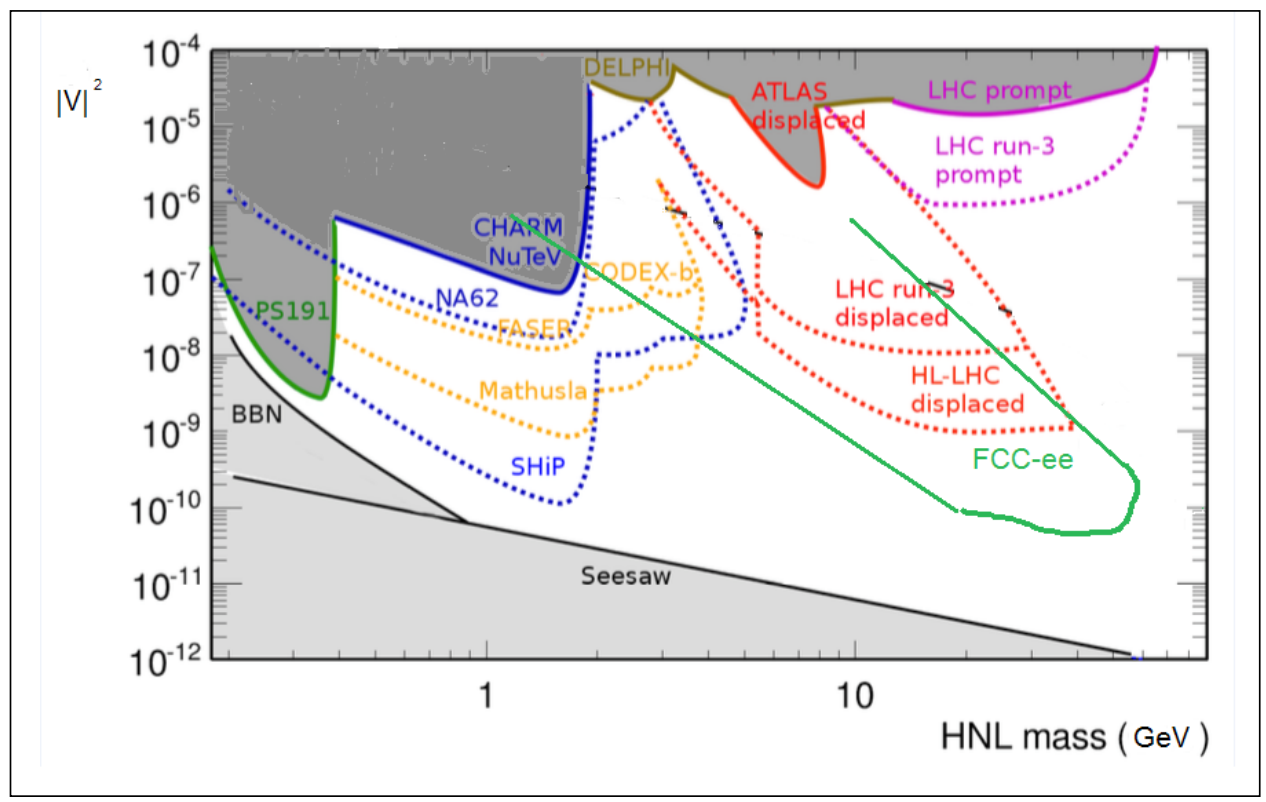

Figure 3. Exclusion plots of current and future experiments in the plane of mixing angle versus HNL mass. Adapted from previous plots to illustrate the reach of future searches. In gray the current bounds from experiments referenced in the text

unlike the decays occurring within main LHC detectors. Figure 3 summarizes the reach of FASER in the relevant parameter space.

MATHUSLA. Mathusla is a modular large surface detector of dimensions $200 \times 200 \times 20 \mathrm{~m}^{3}$ that would be located directly above an LHC interaction point, Curtin, et al. (2018). The main search is for neutral LLPs that are produced at the LHC IP but have long lifetimes, thus producing a very displaced vertex in the Mathusla detector. In addition, unlike the LHC detectors it would have a small background, can have triggers that are specifically designed for the resulting kinematics of the decay process and in some region of parameter space have a larger acceptance. The strength of the proposal is the combination of the energy frontier of the LHC with a dedicated LLP detector. The detector is essentially a tracker, with good timing resolution that can distinguish the main background, coming from cosmic rays, from the signal. This signal is essentially two well separated charged particles originating from a displaced vertex. The particles can be quarks, gluons or charged leptons.

SHIP. The SHIP experiment is proposed in the Beam Dump Facility at CERN, with on the order of $2 \times 10^{20}$ protons delivered on target over a 5 year period, Alekhin, et al. (2015). As a result, large amounts of D and B mesons will be produced which can then decay to the HNL. The subsequent decay on the HNL will be detected with a spectrometer and calorimeters and muon detectors for particle identification. The spectrometer will have the capability of reconstructing the corresponding decay vertex.

FCC-ee. Among the proposals for future colliders an option is the FCC-ee, Blondel, et al. (2016). This facility is a high-luminosity, high-precision e+e- circular collider envisioned in a new 80-100 $\mathrm{km}$ tunnel in the Geneva area, with a centre-of-mass energy from 90 to $400 \mathrm{GeV}$. At the $\mathrm{Z}$ peak, the design luminosity would be $4.6 \times 10^{36}$ $\mathrm{cm}^{-2} \mathrm{~s}^{-1}$, while for $\mathrm{HZ}$ production the luminosity would be $1.6 \times 10^{35} \mathrm{~cm}^{-2} \mathrm{~s}^{-1}$.

Figure 3 presents the current and future exclusion limits of the diverse collider and fixed target /beam dump experiments that have been discussed in this paper. The exclusion lines for future experiments assume mixing with only one right-handed neutrino and zero background.

It is quite impressive to see the enormous coverage of current allowed parameter space, in the $|\mathrm{V}|^{2}-\mathrm{M}_{\mathrm{N}}$ plane, that can be achieved through these experimental efforts. NA62 and LHC experiments currently provide the strongest constraints for masses above the kaon mass scale, placing upper bounds on the mixing angle squared on the order of $10^{-7}$ for masses below the D-meson mass and at most $210^{-6}$ for the displaced vertex mass range 5-10 GeV.

\section{Conclusions}

The possibilities of discovering new physics that is given by weakly interacting long lived particles has been discussed in the context of accelerator-based experiments. For the case of heavy neutral leptons over many decades a wide variety of experiments have provided exclusion regions in the parameter space of the active-sterile mixing angle and the mass of the HNL. Without a doubt, a number of exciting experiments and new proposals are sensitive to discovering LLP both in the energy frontier and the intensity frontier. SHIP will provide more than two orders of magnitude increased sensitivity to the mixing angle squared compared to NA62, for $M_{N}$ in the range 1-2 GeV. FCC-ee could also 
provide a two order of magnitude improvement in the mixing angle upper bound for masses of the right-handed neutrino in the range $10-30 \mathrm{GeV}$, compared to the LHC reach of displaced vertices. The prospects we have discussed can extend the sensitivity reach over more than one or two orders of magnitude for a specific range of masses of the new particles. For the novel signature of a displaced vertex the proposals consider setups to have none or very small backgrounds compared to the LHC.

\section{Conflict of interest}

None to declare

\section{References}

Aaboud, M., et al. (2019c). ATLAS CN, Observation of electroweak WZ boson pair production in association with two jets in $\$ p p \$$ collisions at $\backslash$ sqrt $\{\mathrm{s}\}=13 \mathrm{TeV}$ with the ATLAS detector," Phys. Lett. B 793, 469 (2019). doi:10.1016/j. physletb.2019.05.012

Aaboud, M., et al. (2019d). ATLAS Collaboration, https:// atlas.web.cern.ch/Atlas/GROUPS/PHYSICS/ CombinedSummaryPlots/EXOTICS/ATLAS_Exotics Summary/ATLAS_Exotics_Summary.png, accessed Sept 30, 2019.

Aad, G., et al. (2019a). ATLAS CN, The ATLAS collaboration,Luminosity determination in $\$ p p \$$ collisions at $\$ \backslash$ $\operatorname{sqrt}\{\mathrm{s}\}=13 \$ \mathrm{TeV}$ using the ATLAS detector at the LHC, ATLAS-CONF-2019-021.

Aad, G., et al. (2012). ATLAS CNATLAS Collaboration, Observation of a new particle in the search for the Standard Model Higgs boson with the ATLAS detector at the LHC," Phys. Lett. B 716, 1 (2012). doi:10.1016/j.physletb.2012.08.020

Aad, G., et al. (2015). ATLAS and CMS CN, ATLAS and CMS Collaborations, Combined Measurement of the Higgs Boson Mass in pp Collisions at $\backslash$ sqrt $\{\mathrm{s}\}=7$ and $8 \mathrm{TeV}$ with the ATLAS and CMS Experiments," Phys. Rev. Lett. 114, 191803 (2015). doi:10.1103/PhysRevLett.114.191803

Aad, G., et al. (2016). ATLAS and CMS CN, ATLAS and CMS Collaborations, Measurements of the Higgs boson production and decay rates and constraints on its couplings from a combined ATLAS and CMS analysis of the LHC pp collision data at $\backslash$ sqrt $\{\mathrm{s}\}=7$ and $8 \mathrm{TeV}$," JHEP 1608, 045 (2016). doi:10.1007/JHEP08(2016)045

Aad, G., et al. (2015). ATLAS Collaboration Search for heavy Majorana neutrinos with the ATLAS detector in pp collisions at $s \sqrt{ }=8 \mathrm{~s}=8 \mathrm{TeV}$ - JHEP 1507 (2015) 162 arXiv:1506.06020 [hep-ex] CERN-PH-EP-2015-070

Aad, G., et al. (2019b). ATLAS Collaboration, Search for heavy neutral leptons in decays of \$W $\$$ bosons produced in 13 TeV \$pp\$ collisions using prompt and displaced signatures with the ATLAS detector, arXiv:1905.09787 [hep-ex].

Aaij R, et al. (2017). LHCb Collaboration Search for massive long-lived particles decaying semileptonically in the LHCb detector - Eur.Phys.J. C77 (2017) no.4, 224 arXiv:1612.00945 [hep-ex] CERN-EP-2016-283, LHCBPAPER-2016-047

Abada A, Davidson S, Josse-Michaux FX, Losada M, Riotto A. (2006). Flavor issues in leptogenesis, JCAP 0604, 004 (2006). doi:10.1088/1475-7516/2006/04/004.
Abreu, P., et al. (1997). DELPHI Collaboration Search for neutral heavy leptons produced in Z decays - Z.Phys. C74 (1997) 57-71, Erratum: Z.Phys. C75 (1997) 580 CERNPPE-96-195.

Achard, P., et al. (2001). L3 Collaboration Search for heavy isosinglet neutrino in $e+e-\mathrm{e}+\mathrm{e}-$ annihilation at LEP Phys. Lett. B517 (2001) 67-74 hep-ex/0107014 CERNEP-2001-045.

Adriani, O., et al. (1992). L3 Collaboration Search for isosinglet neutral heavy leptons in Z0 decays - Phys.Lett. B295 (1992) 371-382 CERN-PPE-92-164

Aguilar-Arevalo A, et al. (2015). PIENU Collaboration, Phys. Rev. Lett. 115 (2015) 071801.

Akhmedov E Kh, Rubakov VA, Smirnov AYu. (1998). Baryogenesis via neutrino oscillations Phys.Rev.Lett. 81 (1998) 1359-1362 hep-ph/9803255 IC-98-22, INR-98-14-T

Alehkin S., et al. (2015). A facility to Search for Hidden Particles at the CERN SPS: the SHiP physics case, Rept. Prog. Phys. 79 (2016) 124201, [1504.04855].

Alimena J., et al. (2019). The LHC Long-Lived Particle Community, Searches for long-lived particles beyond the Standard Model at the Large Hadron Collider, http://arxiv.org/ abs/1903.04497

Ariga A., et al. (2018). FASER collaboration, FASER's Physics Reach for Long-Lived Particles, arXiv:1811.12522.

Artamonov A.V., et al. (2015). E949 Collaboration, Search for heavy neutrinos in $K+\rightarrow \mu+v H \mathrm{~K}+\rightarrow \mu+v \mathrm{H}$ decays - Phys.Rev. D91 (2015) no.5, 052001, Erratum: Phys. Rev. D91 (2015) no.5, 059903 arXiv:1411.3963 [hep-ex] FERMILAB-PUB-14-609-E

Asaka T, Shaposhnikov M. (2005). The nuMSM, dark matter and the baryon asymmetry of the Universe, Phys.Lett. B620 (2005) 17-26.DOI: 10.1016/j.physletb.2005.06.020

Astier, P., et al. (2001). NOMAD Collaboration Search for heavy neutrinos mixing with tau neutrinos - Phys.Lett. B506 (2001) 27-38 hep-ex/0101041 CERN-EP-2001-005

Badier, J., et al. (1986). NA3 Collaboration Direct Photon Production From Pions and Protons at $200-\{\mathrm{GeV}\} / c \mathrm{c}-$ Z.Phys. C31 (1986) 341 CERN-EP-85-200

Baranov, S.A., et al. (1993). Search for heavy neutrinos at the IHEP-JINR neutrino detector - Phys.Lett. B302 (1993). 336-340 JINR-P1-92-494

Beacham, J., et al. (2019), Physics Beyond Colliders at CERN: Beyond the Standard Model Working Group Report, arXiv:1901.09966 [hep-ex].

Bergsma, F., et al. (1986), CHARM Collaboration A Search for Decays of Heavy Neutrinos in the Mass Range 0.5-\{GeV\} to $2.8-\{\mathrm{GeV}\}$ - Phys.Lett. 166B (1986) 473-478 CERNEP-85-190Bergstrom (2012), Bergstrom, L., Dark Matter Evidence, Particle Physics Candidates and Detection Methods, Annalen Phys. 524 (2012) 479-496.

Bernardi, G., et al. (1988). Further Limits On Heavy Neutrino Couplings - Phys.Lett. B203 (1988). 332-334 CERNEP/87-23.

Blondel, A., et al. (2016). FCC-ee study Team collaboration, Search for Heavy Right Handed Neutrinos at the FCCee, Nucl. Part. Phys. Proc. 273-275 (2016). 1883-1890, [1411.5230].

Bhupal, D.P.S., et al. (2018), Flavor effects in leptogenesis Int.J.Mod.Phys. A33 (2018). 1842001 arXiv:1711.02861 [hep-ph] and references therein. 
Boyarsky, A., Ruchyarsky, O, Shaposhnikov M. (2009), The role of sterile neutrinos in cosmology and astrophysics, Ann.Rev.Nucl.Part.Sci. 59 (2009) 191-214.

Chatrchyan, et al. (2012). CMS CN, CMS Collaboration, observation of a new boson at a mass of $125 \mathrm{GeV}$ with the CMS experiment at the LHC,Phys. Lett. B 716, 30 (2012). doi:10.1016/j.physletb.2012.08.021

Cooper-Sarkar, A.M., et al. (1986). WA66 Collaboration Search for Heavy Neutrino Decays in the $\{$ BEBC $\}$ Beam Dump Experiment - Phys.Lett. 160B (1985) 207-211 CERNEP-85-104.

Cortina Gil, E., et al. (2019). NA62 collaboration, Searches for lepton number violating $\$ \mathrm{~K}^{\wedge}+\$$ decays, " arXiv: 1905.07770 [hep-ex.]

Curtin, D., et al. (2018). Long-Lived Particles at the Energy Frontier: The MATHUSLA Physics Case, arXiv:1806.07396.

Davidson S., Nardi E., Nir Y. (2008). Leptogenesis -. Phys.Rept. 466 (2008) 105-177 arXiv:0802.2962 [hep-ph]

Drewes M., et al. (2018). ARS Leptogenesis - Int.J.Mod.Phys. A33 (2018) no.05n06, 1842002 arXiv:1711.02862 [hep-ph]

Drewes M., Giammanco A., Hajer J., Lucente, M. (2019). Long Lived Particles Searches in Heavy Ion Collisions at the LHC,arXiv:1905.09828 [hep-ph].

Esteban, I., et al. (2017). Updated fit to three neutrino mixing: exploring the accelerator-reactor complementarity, JHEP 1701 (2017) 087, doi:10.1007/JHEP01(2017)087

Feng, J.L., et al. (2018). ForwArd Search ExpeRiment at the LHC, Phys. Rev. D97 (2018) 035001, [1708.09389].

Fukugita, M. and Yanagida, H. (1986). Baryogenesis Without Grand Unification - Phys.Lett. B174 (1986) 45-47 RIFP641.

Gallas, E., et al. (1995). FMMF Collaboration Search for neutral weakly interacting massive particles in the Fermilab Tevatron wide band neutrino beam - Phys.Rev. D52 (1995) 6-14.

Gronau M., Leung C.N., Rosner, J. (1984). Extending limits on neutral heavy leptons, Phys. Rev. D 29, 1984, 2539.

Hernandez, P. (2015). Neutrino Physics, CERN Yellow Report CERN 2015-005, pp 85-142.

Hernandez P., Kekic M., Lopez-Pavon J., Racker J., Salvado J. (2016). Testable Baryogenesis in Seesaw Models, JHEP 1608 (2016) 157 arXiv:1606.06719 [hep-ph].

Khachatryan V., et al. (2015). CMS Collaboration Search for heavy Majorana neutrinos in $\mu \pm \mu \pm+\mu \pm \mu \pm+$ jets events in proton- proton collisions at $s \sqrt{ } \mathrm{s}=8 \mathrm{TeV}$ - Phys.Lett. B748 (2015) 144-166 arXiv:1501.05566 [hep-ex] CMS-EXO-12-057, CERN-PH-EP-2015-001.

Lazzeroni C., et al. (2017). NA62 Collaboration Search for heavy neutrinos in $K+\rightarrow \mu+v \mu \mathrm{K}+\rightarrow \mu+v \mu \quad$ decays-Phys.Lett. B772 (2017) 712-718 arXiv:1705.07510 [hep-ex] CERNEP-2017-114, Search for heavy neutral lepton production in K+decays, Phys. Lett. B778 (2018) 137-145, [1712.00297]

Lee L., Ohm C., Soffer A., Yu T.T. (2019). Collider Searches for Long-Lived Particles Beyond the Standard Model, JPPNP 3695 (2019).

Majorana, E. (1937). Teoria simmetrica dell'elettrone e del positrone, Nuovo Cim.\\{〈bf 14\}, 171 (1937). doi:10.1007/ BF02961314

Nardi E., Nir Y., Racker, J., Roulet, E. (2006). ' 'The Importance of flavor in leptogenesis," JHEP 0601, 164 (2006). doi:10.1088/1126-6708/2006/01/164.

Shaposhnikov, M. (2008). The nuMSM, leptonic asymmetries, and properties of singlet fermions - JHEP 0808 (2008) 008 arXiv:0804.4542 [hep-ph]

Sirunyan A., et al. (2019a). CMS CN, CMS Collaboration, Measurement of electroweak WZ boson production and search for new physics in $\mathrm{WZ}+$ two jets events in pp collisions at $s \sqrt{ }=\mathrm{S}=13 \mathrm{TeV}$ CMS Collaboration, Phys.Lett. B795 (2019) 281-307. doi: 10.1016/j.physletb.2019.05.042

Sirunyan A., et al. (2019b). CMS CN, CMS Collaboration, https://twiki.cern.ch/twiki/pub/CMSPublic/ SummaryPlotsEXO13TeV/EXO_barchart_Jan19.svg accessed, Sept 30, 2019.

Sirunyan A., et al. (2018). CMS Collaboration,Search for heavy neutral leptons in events with three charged leptons in proton-proton collisions at $\$ \backslash$ sqrt $\{\mathrm{s}\}=\$ 13 \mathrm{TeV}$," Phys. Rev. \Lett. \\{|bf 120\}, no. 22, 221801 (2018) doi:10.1103/ PhysRevLett.120.221801

Vaitaitis U.K., et al. (1999). NuTeV and E815 Collaborations Search for neutral heavy leptons in a high-energy neutrino beam - Phys.Rev.Lett. 83 (1999) 4943-4946 hepex/9908011 FERMILAB-PUB-99-223-E.

Vilain P., et al. (1995). CHARM II Collaboration Search for heavy isosinglet neutrinos - Phys.Lett. B343 (1995) 453-458, Phys. Lett. B351 (1995) 387-392 CERN-PPE-94-16801422-9. doi:10.1016/0370-2693(94)

Zwicky F. (1933). "Die Rotverschiebung von extragalaktischen Nebeln", Helvetica Physica Acta, 6: 110-127. 